\title{
SMC1
}

\section{Iterative methods for the reconstruction of LINC-NIRVANA images}

\author{
Anconelli B., Bertero M., Boccacci P. \\ INFM and DISI, Università di Genova, Via Dodecaneso 35, 16146 Genova, Italy \\ +39(10) 3536610, +39 (10)3536699, surname@disi.unige.it \\ Carbillet M., Lanteri H. \\ UMR 6525 Astrophysique, Université de Nice - Sophia Antipolis, Faculté des \\ Sciences - Parc Valrose, 06108 Nice Cedex 02, France \\ +33(49)2076340, marcel.carbillet@unice.fr, Henri.LANTERI@unice.fr
}

\begin{abstract}
The Large Binocular Telescope (LBT) will require routinely the use of multipleimage deconvolution methods. We present the methods and software we have developed for this data reduction problem. (C) 2005 Optical Society of America

OCIS codes: (100.3020) Image reconstruction-restoration ; (100.2960) Image analysis
\end{abstract}

\section{Introduction}

The Large Binocular Telescope (LBT) will consist of two $8.4 \mathrm{~m}$ mirrors on a common mount, with a spacing of $14.4 \mathrm{~m}$ between the centres of the two mirrors, so that a maximum baseline of $22.8 \mathrm{~m}$ will be available. LBT will be equipped with a Fizeau interferometer, denoted as LINC/NIRVANA, (LN) which will be the result of a German-Italian collaboration. First light of LBT is scheduled for September 2004, second light for November 2005, and the first LN light is foreseen for July 2006.

The interferometric technique used in LN will provide direct imaging with the resolution of a $22.8 \mathrm{~m}$ telescope in the direction of the baseline and of a $8.4 m$ telescope in the orthogonal direction. Since resolution is not uniform over the field, several images of the same scientific object must be acquired with different orientations of the baseline and they must be suitably processed in order to get a unique image with a uniform resolution over the field. Therefore imaging by LN will require routinely the use of multiple-images deconvolution methods if the goal is a unique image with the resolution of a $22.8 \mathrm{~m}$ telescope.

According to our experience and taking into account the features of astronomical images which can contain objects with different angular scales, very different magnitudes and therefore also very different local SNR, we think that two different classes of deconvolution methods will be required for LN imaging.

- General purpose methods, computationally efficient as far as possible, to be used just after observation, in order to identify the main features of the observed object. The accelerated version of OSEM we have developed could be used for this task.

- Dedicated methods, developed for particular classes of objects with particular features. Obviously each astronomer can use his own method for the kind of objects he is investigating. However, due to the novelty of LN imaging, it could be useful to develop specific methods at least for a few important classes of astronomical objects, such as objects with very high dynamic range, faint objects and unresolved stellar objects, with angular separations not much smaller than the diffraction limit.

\section{The mathematical approach}

In a series of recent papers Lanteri et al. $[4,5]$ proposed a general approach to the design of iterative methods for the minimization of functionals of the following type:

$$
J_{\mu}(\mathbf{f} ; \mathbf{g})=J_{0}(\mathbf{f} ; \mathbf{g})+\mu J_{R}(\mathbf{f})
$$




\section{SMC1}

with the additional constraints of non negativity and flux conservation. In this equations $J_{0}(\mathbf{f} ; \mathbf{g})$ is the functional measuring the discrepancy between the computed images associated to $\mathbf{f}$ and the detected images $\mathrm{g}$ and $J_{R}(\mathbf{f})$ is a regularization functional while $\mu$ is the regularization parameter.

The basic idea in the approach of Lanteri et al. relies on the following decomposition of the gradient of the functional $J_{\mu}(\mathbf{f})$ :

$$
-\nabla J_{\mu}(\mathbf{f} ; \mathbf{g})=\mathbf{U}_{\mu}(\mathbf{f} ; \mathbf{g})-\mathbf{V}_{\mu}(\mathbf{f} ; \mathbf{g})
$$

where $\mathbf{U}_{\mu}(\mathbf{f} ; \mathbf{g})$ and $\mathbf{V}_{\mu}(\mathbf{f} ; \mathbf{g})$ are positive arrays depending on $\mathbf{f}$. Then the algorithm is as follows:

$$
\mathbf{f}^{(k+1)}=\mathbf{f}^{(k)} \frac{\mathbf{U}_{0}\left(\mathbf{f}^{(k)} ; \mathbf{g}\right)+\mu \mathbf{U}_{R}\left(\mathbf{f}^{(k)}\right)}{\mathbf{V}_{0}\left(\mathbf{f}^{(k)} ; \mathbf{g}\right)+\mu \mathbf{V}_{R}\left(\mathbf{f}^{(k)}\right)}
$$

where the very simple dependence of the algorithm on the regularization parameter $\mu$ is shown.

If we assume to have p images acquired with LBT, then the functional $J_{0}\left(\mathbf{f} ; \mathbf{g}_{j}\right)$ is given by:

$$
J_{0}\left(\mathbf{f} ; \mathbf{g}_{j}\right)=\sum_{j=1}^{p} \sum_{m, n=0}^{N-1}\left\{\mathbf{g}_{j}(m, n) \ln \frac{\mathbf{g}_{j}(m, n)}{\left(A_{j} \mathbf{f}\right)(m, n)+\mathbf{b}_{j}}+\left[\left(A_{j} \mathbf{f}\right)(m, n)+\mathbf{b}_{j}-\mathbf{g}_{j}(m, n)\right]\right\} ;
$$

In the case of $\mu=0$, one re-obtains from Eq. 3 the multiple-image Richardson-Lucy method. In the case of a regularization functional with $\mathbf{U}_{R}(\mathbf{f})=0$, we get:

$$
\mathbf{f}^{(k+1)}=\frac{1}{p} \frac{\mathbf{f}^{(k)}}{\mathbf{1}+\mu \mathbf{V}_{R}\left(\mathbf{f}^{(k)}\right)} \sum_{j=1}^{p} A_{j}^{T} \frac{\mathbf{g}_{\mathbf{j}}}{A_{j} \mathbf{f}^{(k)}+\mathbf{b}_{\mathbf{j}}} .
$$

We implemented accelerated version of these algorithms, based on the approach of OSEM.

\section{Results}

We performed several simulations about the reconstruction of a diffuse object and of a high-dynamic one that shown in Fig. 2. We used a set of ideal PSF and different sets of AO-corrected PSF.

\subsection{Reconstruction of diffuse objects}

We tested RLM with the image of a Spirograph nebulae (see Fig. 1) with differents magnitude (14, 15, 16); we compute the RMS and the optimal number of iterations and we noticed that increasing the SNR the reconstruction error slows down while the number of iteration increases. For example with $\mathrm{m}=14$ the minimum of the reconstruction error is $6.6 \%$ in 544 iterations and for $\mathrm{m}=16$ we reached a $12.5 \%$ in 198 iterations.
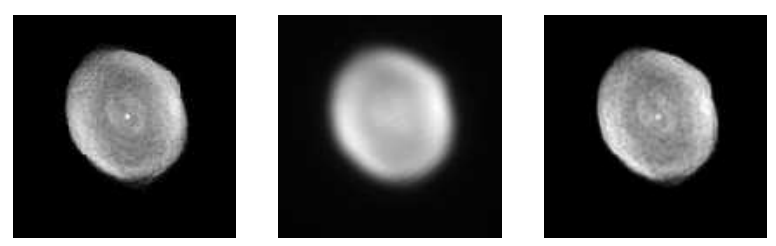

Fig. 1. Spirograph nebulae. Left box: the object; central box: one of the images; right box: reconstructed image 


\section{SMC1}
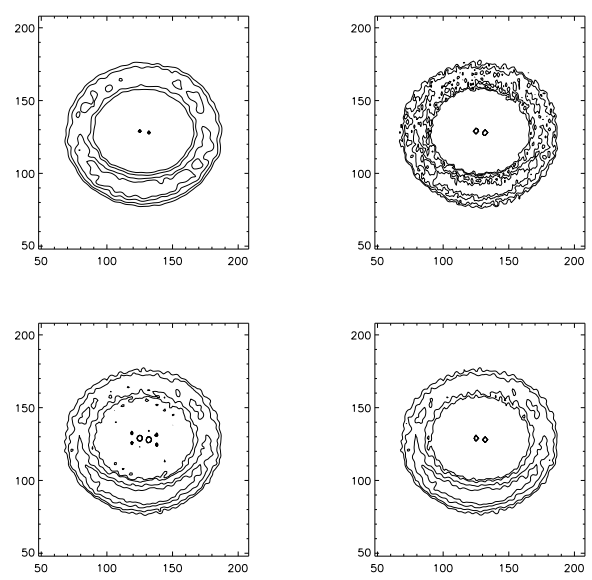

Fig. 2. Representation of the ring by means of level curves. The values corresponding to the plotted curves are given in the text. Upper-left panel: the object; upper-right: the reconstruction provided by RLM; lower-left: reconstruction with convex regularization; lower-right: reconstruction with non-convex regularization.

\subsection{Reconstruction of images with high dynamic range}

The accurate reconstruction of objects such as that shown in Fig. 2 is a difficult problem [8]. In this case the important feature is that there is a large gap between the intensity of bright $\left(10^{9}\right.$ photons $)$ and faint objects (4000 photons) and that these different objects are located in different image domains. We have tested the algorithms above on the object of Fig. 2 using a regularization functional such as:

$$
J_{R}(f)=\sum_{m, n=0}^{N-1} \frac{\eta^{2} f^{2}(m, n)}{\eta^{2}+f^{2}(m, n)}
$$

where $\eta$ is a thresholding parameter. To give a more precise idea of the accuracy of the reconstructions of the ring provided by the different methods, in Fig. 3 we represent the ring in term of level curves. Finally, all the functionals provide a good reconstruction of the binary, with an error in magnitude of about $0.5 \%$.

\section{References}

1. Bertero M., and Boccacci P. (2000) Application of the OS-EM method to the restoration of LBT images, Astron. Astrophys. Suppl. Ser, 144, 181-186.

2. Correia S., Carbillet M., Boccacci P., Bertero M., and Fini L. (2002) Restoration of interferometric images - I. The software package AIRY. Astron. Astrophys., bf 387, 733-743.

3. Carbillet M., Correia S., Boccacci P., and Bertero M. (2002) Restoration of interferometric images - II. The case-study of the Large Binocular Telescope, Astron. Astrophys., 387, 744-757.

4. Lanteri H., Roche M., Cuevas O., and Aime C. (2001) A general method to devise maximum-likelihood signal restoration multiplicative algorithms with non-negativity constraints, Signal Process., 81, 945-974.

5. Lanteri H., Roche M., and Aime C. (2002) Penalized maximum likelihood image restoration with positivity constraints: multiplicative algorithms, Inverse Problems, 18, 1397-1419.

6. Anconelli B., Bertero M., Boccacci P., and Carbillet M. (2004) Restoration of interferometric images - IV. An algorithm for super-resolution of stellar systems, submitted to Astron. Astrophys..

7. Anconelli B., Bertero M., Boccacci P., Carbillet M., and Lanteri H. (2004) Restoration of interferometric images III. Efficient Richardson-Lucy methods for LINC/NIRVANA data reduction. (to appear in A\&A)

8. Bertero M., Anconelli B., Boccacci P., Carbillet M., and Lanteri H. (2004) Iterative methods for the reconstruction of astronomical images with high dynamic range. (submitted to JCAM) 